\title{
Global stability for a new predator-prey model with cross-dispersal among patches based on graph theory
}

\section{Yang $\mathrm{GaO}^{1 *}$ (1)}

"Correspondence:

gy19790607@163.com

'Department of Mathematics,

Daqing Normal University, Daqing,

163712, China

\section{Springer}

\begin{abstract}
In this paper, cross-dispersal is considered in a predator-prey model with a patchy environment. A new predator-prey model with cross-dispersal among patches is constructed. A new cross-dispersal matrix is established by the coupling relationship between vertices. First, an existence theorem of the positive equilibrium for the new model is obtained. Secondly, based on the idea of constructing Lyapunov functions and a graph-theoretical approach for coupled systems, sufficient conditions that the positive equilibrium of the new model is globally asymptotically stable in $R_{+}^{2 n}$ are derived on a network with strongly connected graphs. Thirdly, based on the theory of asymptotically autonomous systems, Lyapunov functions method and graph theory, a stability theorem for the positive equilibrium of the new model is established on a complex network without strongly connected graphs. Finally, two examples are given to illustrate main results.
\end{abstract}

Keywords: Cross-dispersal; Coupled model; Global stability; Predator-prey model; Patches

\section{Introduction}

In the literature of predator-prey systems, it is an interesting problem to consider a patchy environment. In reality, species always disperse from one patch to another patch. It is an important topic to consider the population dynamics of multi-patch predator-prey models with dispersal. Dispersal among predators or among various prey has been studied by researchers for many years. Dispersal among predators is called self-dispersal of predators, while dispersal among various prey is called self-dispersal of prey. The dynamics of predator-prey models with self-dispersal have been studied by researchers in recent years. Many researchers devoted more time to discussing self-dispersal of prey, self-dispersal of predators and self-dispersal for both predators and prey of predator-prey models [1-6]. Self-dispersal of prey among $n$ patches was considered in Refs. [1-3]. However, the models presented in Refs. [1-3] were slightly different. One was to model and study multi-patch periodic predator-prey systems [1]. Another was to consider multi-patch predator-prey systems without considering the periodic character [2]. The other was to study multipatch predator-prey systems with a Holling type-II functional response [3]. Predator-

(c) The Author(s) 2021. This article is licensed under a Creative Commons Attribution 4.0 International License, which permits use, sharing, adaptation, distribution and reproduction in any medium or format, as long as you give appropriate credit to the original author(s) and the source, provide a link to the Creative Commons licence, and indicate if changes were made. The images or other third party material in this article are included in the article's Creative Commons licence, unless indicated otherwise in a credit line to the material. If material is not included in the article's Creative Commons licence and your intended use is not permitted by statutory regulation or exceeds the permitted use, you will need to obtain permission directly from the copyright holder. To view a copy of this licence, visit http://creativecommons.org/licenses/by/4.0/. 
prey systems with self-dispersal of predators between two patches were studied in [4]. Predator-prey models with $n$ patches and self-dispersal for both predators and prey were investigated in $[5,6]$.

Because of the close relationship among species in different patches, cross-dispersal should be considered in the real environment. In fact, predator dispersal can affect prey density, while prey dispersal also can affect predator density. Hence, it is necessary to study the dynamics of the predator-prey model with cross-dispersal among all patches. Recently, cross-dispersal was used to study multi-group models (see [7]). Based on graph theory and Lyapunov functions method, the dynamics of a general multi-group model with cross-dispersal were given in [7]. To the best of the author's knowledge, few researchers have focused on the dynamics of predator-prey models with cross-dispersal. Hence, it is important to discuss the problem in this paper.

Based on graph theory, a systematic approach to construct global Lyapunov functions for coupled systems was developed by the authors of [2]. Much work had been done in order to apply this method to many areas $[1-3,6,8-11]$. The systematic approach was based on the assumption that the network was strongly connected. However, to the best of the author's knowledge, graphs without strong connectedness are universal in reality. Due to dealing with large-scale complex networks without strong connectedness, a hierarchical method and a hierarchical algorithm were proposed in [12]. Based on the hierarchical algorithm and the theory of asymptotically autonomous systems, stability theorems for a new fractional-order coupled system on a network without strong connectedness were obtained in [13].

Although predator-prey models based on ordinary differential equations (ODE) have been discussed for many years, to the best of the author's knowledge, cross-dispersal was not considered in the predator-prey model based on ODE by researchers. In fact, crossdispersal is reasonable and applicable for patchy environment. The construction for the new predator-prey model with cross-dispersal in a patchy environment is interesting and can be widely applied to the ecological field. In order to fill this gap, a new predatorprey model with cross-dispersal is constructed in this paper. To the best of the author's knowledge, the new predator-prey model with cross-dispersal constructed herein has not been proposed in any other literature. The new cross-dispersal matrix presented here has not been established by any other researcher. Based on the method of graph theory and Lyapunov theory, global stability theorems of the positive equilibrium are established. Innovative points are listed as follows:

1. Cross-dispersal is introduced into the predator-prey model in a patchy environment. A new predator-prey model is established.

2. A new cross-dispersal matrix is established by the coupling relationship between vertices.

3. Based on the idea of graph theory, a global stability theorem for the positive equilibrium is established on a network with strongly connected graphs.

4. Based on the theory of asymptotically autonomous systems and graph theory, a global stability theorem for the positive equilibrium is established on a network with strongly connected components, but without strongly connected graphs.

This paper is organised as follows. Preliminary results are introduced in Sect. 2. In Sect. 3, the main results are obtained, and examples are presented in Sect. 4. Finally, conclusions and outlook are outlined in Sect. 5. 


\section{Preliminaries}

In this section, some definitions and theorems are listed that will be used in the later sections (see $[2,12,13])$. We denote a weighted digraph as $(G, A)$. A digraph $G$ is strongly connected if, for any pair of distinct vertices, there exists a directed path from one to the other. A weighted digraph $(G, A)$ is strongly connected if and only if the weight matrix $A$ is irreducible. Furthermore, a strongly connected component $H$ of a digraph $G$ is defined as follows: if the subgraph $H$ is strongly connected and for any vertex $k \notin V(H)$, the subgraph that consists of the vertex set $V(H) \cup\{k\}$ is not strongly connected, then $H$ is a strongly connected component.

Lemma 2.1 ([2]) Assume $n \geq 2$. Let $c_{i}$ be given in Proposition 2.1 of Ref. [2]. Then, the following identity holds:

$$
\sum_{i, j=1}^{n} c_{i} a_{i j} F_{i j}\left(x_{i}, x_{j}\right)=\sum_{Q \in \mathrm{Q}} w(Q) \sum_{(s, r) \in E\left(C_{\mathrm{Q}}\right)} F_{r s}\left(x_{r}, x_{s}\right) .
$$

Here, $F_{i j}\left(x_{i}, x_{j}\right), 1 \leq i, j \leq n$, are arbitrary functions, $\mathrm{Q}$ is the set of all spanning unicyclic graphs of $(G, A), w(Q)$ is the weight of $Q$, and $C_{\mathrm{Q}}$ denotes the directed cycle of $\mathrm{Q}$.

If $(G, A)$ is balanced, then

$$
\sum_{i, j=1}^{n} c_{i} a_{i j} F_{i j}\left(x_{i}, x_{j}\right)=\frac{1}{2} \sum_{Q \in \mathrm{Q}} w(Q) \sum_{(j, i) \in E\left(C_{\mathrm{Q}}\right)}\left[F_{i j}\left(x_{i}, x_{j}\right)+F_{j i}\left(x_{j}, x_{i}\right)\right] .
$$

\section{Main results}

Based on cross-dispersal, the new predator-prey model is constructed as follows:

$$
\left\{\begin{array}{l}
\dot{x_{i}}=x_{i}\left(r_{i}-b_{i} x_{i}-e_{i} y_{i}\right)-\sum_{j=1}^{n} k_{i j}^{x y} y_{j} s_{i j} x_{i}, \\
\dot{y}_{i}=y_{i}\left(-\gamma_{i}-\delta_{i} y_{i}+\varepsilon_{i} x_{i}\right)+\sum_{j=1}^{n} k_{i j}^{y x} x_{j} z_{i j} p_{i j} y_{i}, \quad i=1, \ldots, n .
\end{array}\right.
$$

Here, $x_{i}, y_{i}$ denote the densities on patch $i$ for various prey and predators, respectively. Model parameters $b_{i}, \delta_{i}, e_{i}, \varepsilon_{i}$ are all positive constants. $r_{i}$ and $\gamma_{i}$ are non-negative constants. $k_{i j}^{x y}$ denotes the dispersal rate of predators from patch $j$ to patch $i . s_{i j} x_{i}$ denotes functional response of predators that disperse from patch $j$ to patch $i . k_{i j}^{y x}$ denotes the dispersal rate of various prey from patch $j$ to patch $i . p_{i j} x_{j}$ denotes the functional response of predators on patch $i$ to various prey that disperse from patch $j$ to patch $i . z_{i j}$ denotes the conversion rate of various prey that come from patch $j$ and are preyed on by predators on patch $i$. The meanings of the above parameters are listed as Table 1 .

Based on the Volterra predator-prey model [2], the new model constructed is reasonable. Dispersal assumptions are reasonable from modelling. Predator-prey systems with $n$ patches can be studied and explained from the biological viewpoint, any prey dispersed to this patch can be preyed on by predators on this patch. Furthermore, any prey can be preyed on not only by predators on this patch, but also by predators dispersed to this patch. In other words, any prey have a positive effect on the predator when prey disperse to a predator's population. Prey dispersed from patch $j$ can be preyed on by predators on patch $i$. Furthermore, the predator population on patch $i$ will increase, while predators have a negative effect on prey when predators disperse to a prey's population. Predators 
Table 1 Parameters used in the new model

\begin{tabular}{ll}
\hline Symbol & Definition \\
\hline$r_{i}$ & birth rate for prey population on patch $i$ \\
$e_{i} x_{i}$ & functional response of predators on patch $i$ \\
$\gamma_{i}$ & death rate for predator population on patch $i$ \\
$\frac{\varepsilon_{i}}{e_{i}}$ & conversion rate of prey on patch $i$ \\
$\frac{r_{i}}{b_{i}}$ & environmental capacity for prey population on patch $i$ \\
$\frac{\gamma_{i}}{\delta_{i}}$ & environmental capacity for predator population on patch $i$ \\
$k_{i j}^{y y}$ & dispersal rate of predators from patch $j$ to patch $i$ \\
$k_{i j}^{y x}$ & dispersal rate of prey from patch $j$ to patch $i$ \\
$s_{i j} x_{i}$ & functional response of predators that disperse from patch $j$ to patch $i$ \\
$p_{i j} x_{j}$ & functional response of predators on patch $i$ to prey that disperse from patch $j$ to patch $i$ \\
$z_{i j}$ & conversion rate of prey that come from patch $j$ and are preyed on by predators on patch $i$ \\
\hline
\end{tabular}

dispersed from patch $j$ can prey on patch $i$. In addition, the prey population on patch $i$ will decrease. When $i=j$, we assume $k_{i i}^{x y}=k_{i i}^{y x}=0$ in the new model (1).

For simplicity, let

$$
-k_{i j}^{x y} s_{i j}=d_{i j}^{x y}, \quad k_{i j}^{y x} z_{i j} p_{i j}=d_{i j}^{y x} .
$$

The above model (1) is transformed into the following model:

$$
\left\{\begin{array}{l}
\dot{x}_{i}=x_{i}\left(r_{i}-b_{i} x_{i}-e_{i} y_{i}\right)+\sum_{j=1}^{n} d_{i j}^{x y} y_{j} x_{i}, \\
\dot{y}_{i}=y_{i}\left(-\gamma_{i}-\delta_{i} y_{i}+\varepsilon_{i} x_{i}\right)+\sum_{j=1}^{n} d_{i j}^{y x} x_{j} y_{i}, \quad i=1, \ldots, n .
\end{array}\right.
$$

The model (2) is equivalent to the next model (3):

$$
\left\{\begin{array}{l}
\dot{x}_{i}=x_{i}\left[\left(r_{i}-b_{i} x_{i}-e_{i} y_{i}\right)+\sum_{j=1}^{n} d_{i j}^{x y} y_{j}\right], \\
\dot{y}_{i}=y_{i}\left[\left(-\gamma_{i}-\delta_{i} y_{i}+\varepsilon_{i} x_{i}\right)+d_{i j}^{y x} x_{j}\right], \quad i=1, \ldots, n .
\end{array}\right.
$$

Remark 3.1 Model (1) is different from the predator-prey model with dispersal that has been studied in recent years. The cross-dispersal is considered in model (1). This means that predators can disperse to a prey population, while prey can also disperse to a predator's population.

Remark 3.2 The biological significance of model (1) is that a patchy environment is formed under the influence of natural conditions or human activities. Predator populations can disperse to other patches to prey, prey species can also disperse to other patches to be preyed on by predators. For example, Eagles prey on rabbits. Rabbits can migrate across different patches, and so can eagles.

Since the above model (1) is equivalent to model (2), we will discuss model (2) in the following sections.

\subsection{The existence of the positive equilibrium for new model (2)}

By the locally Lipschitz character of model (2)'s right-side function and the equivalent model (3), positive solutions' local existence is obvious. Now, we will construct a compact subset to prove the positive solutions' global existence [14-18]. 


\section{Lemma 3.1 If}

$$
\begin{aligned}
& \frac{\left[\varepsilon_{i} b_{i}-\sum_{j=1}^{n}\left(\varepsilon_{i}\left|d_{i j}^{x y}\right|+e_{j}\left|d_{j i}^{y x}\right|\right)\right]}{\varepsilon_{i}^{2}}>0, \\
& \frac{\left[e_{i} \delta_{i}-\sum_{j=1}^{n}\left(e_{i}\left|d_{i j}^{y x}\right|+\varepsilon_{j}\left|d_{j i}^{x y}\right|\right)\right]}{e_{i}^{2}}>0
\end{aligned}
$$

for $i=1,2, \ldots, n$, then there must exist a $N^{*}>0$ such that $G:=\left\{\left(x_{1}, x_{2}, \ldots, x_{n}, y_{1}, y_{2}, \ldots, y_{n}\right) \in\right.$ $\left.R_{+}^{2 n}: \sum_{i=1}^{n}\left(\epsilon_{i} x_{i}+e_{i} y_{i}\right) \leq N^{*}\right\}$ is positively invariant for system (2).

Proof Let $l_{i}=\max \left\{r_{i}, \gamma_{i}\right\}$,

$$
\begin{aligned}
& q_{i}^{1}=\frac{\left[\varepsilon_{i} b_{i}-\sum_{j=1}^{n}\left(\varepsilon_{i}\left|d_{i j}^{x y}\right|+e_{j}\left|d_{j i}^{y x}\right|\right)\right]}{\varepsilon_{i}^{2}}, \\
& q_{i}^{2}=\frac{\left[e_{i} \delta_{i}-\sum_{j=1}^{n}\left(e_{i}\left|d_{i j}^{y x}\right|+\varepsilon_{j}\left|d_{j i}^{x y}\right|\right)\right]}{e_{i}^{2}},
\end{aligned}
$$

and $q_{i}=\frac{1}{2} \min \left\{q_{i}^{1}, q_{i}^{2}\right\}, N=\sum_{i=1}^{n} N_{i}, N_{i}=\epsilon_{i} x_{i}+e_{i} y_{i}$. We have

$$
N^{\prime} \leq \sum_{i=1}^{n}\left(l_{i}-q_{i} N_{i}\right) N_{i} .
$$

Here, we use the Mean Value Inequality with $2 x y \leq x^{2}+y^{2}$.

Furthermore,

$$
N^{\prime} \leq \sum_{i=1}^{n}\left(l_{i}-q_{i} N_{i}\right) N_{i} \leq\left(l-\frac{q}{n} N\right) N
$$

where $l=\max _{i}\left\{l_{i}\right\}, q=\min _{i}\left\{q_{i}\right\}$.

Let $N^{*}=\frac{2 \ln }{q}$. When $N>N^{*}$, we obtain

$$
N^{\prime} \leq \sum_{i=1}^{n}\left(l_{i}-q_{i} N_{i}\right) N_{i} \leq-\frac{1}{2} N \leq 0 .
$$

This means that $G$ is positively invariant. The proof is completed.

Therefore, we have found a compact subset $D=G$. Now, the positive solutions' global existence for model (2) can be obtained as follows:

\section{Lemma 3.2 If}

$$
\begin{aligned}
& \frac{\left[\varepsilon_{i} b_{i}-\sum_{j=1}^{n}\left(\varepsilon_{i}\left|d_{i j}^{x y}\right|+e_{j}\left|d_{j i}^{y x}\right|\right)\right]}{\varepsilon_{i}^{2}}>0, \\
& \frac{\left[e_{i} \delta_{i}-\sum_{j=1}^{n}\left(e_{i}\left|d_{i j}^{y x}\right|+\varepsilon_{j}\left|d_{j i}^{x y}\right|\right)\right]}{e_{i}^{2}}>0
\end{aligned}
$$


for $i=1,2, \ldots, n$, there is a unique solution $Z(t)=\left(x_{1}(t), y_{1}(t), x_{2}(t), y_{2}(t), \ldots, x_{n}(t), y_{n}(t)\right)^{T}$ that is defined for any $t \geq t_{0}$ with $Z\left(t_{0}\right)=Z_{0} \in D$ for model (2).

Note that when $N>N^{*}$,

$$
N^{\prime} \leq-\frac{1}{2} N \leq 0
$$

holds. Therefore, we obtain if $Z\left(t_{0}\right)=Z_{0} \in R_{+}^{2 n}$ and $N\left(Z_{0}\right)>N^{*}$, then

$$
N^{\prime} \leq-\frac{1}{2} N \leq 0
$$

This means that $D:=\left\{\left(x_{1}, x_{2}, \ldots, x_{n}, y_{1}, y_{2}, \ldots, y_{n}\right) \in R_{+}^{2 n}: \sum_{i=1}^{n}\left(\epsilon_{i} x_{i}+e_{i} y_{i}\right) \leq N\left(Z_{0}\right)\right\}$ is positively invariant for system (2). The next lemma is obtained naturally.

Lemma 3.3 If

$$
\begin{aligned}
& \frac{\left[\varepsilon_{i} b_{i}-\sum_{j=1}^{n}\left(\varepsilon_{i}\left|d_{i j}^{x y}\right|+e_{j}\left|d_{j i}^{y x}\right|\right)\right]}{\varepsilon_{i}^{2}}>0, \\
& \frac{\left[e_{i} \delta_{i}-\sum_{j=1}^{n}\left(e_{i}\left|d_{i j}^{y x}\right|+\varepsilon_{j}\left|d_{j i}^{x y}\right|\right)\right]}{e_{i}^{2}}>0
\end{aligned}
$$

for $i=1,2, \ldots, n$, there is a unique solution $Z(t)=\left(x_{1}(t), y_{1}(t), x_{2}(t), y_{2}(t), \ldots, x_{n}(t), y_{n}(t)\right)^{T}$ that is defined for any $t \geq t_{0}$ with $Z\left(t_{0}\right)=Z_{0} \in R_{+}^{2 n}$ for model (2).

Proof Two cases are considered for this lemma. One is that when $N\left(Z_{0}\right) \leq N^{*}, G$ is a required compact and positively invariant set. The other is that when $N\left(Z_{0}\right)>N^{*}, D:=$ $\left\{\left(x_{1}, x_{2}, \ldots, x_{n}, y_{1}, y_{2}, \ldots, y_{n}\right) \in R_{+}^{2 n}: \sum_{i=1}^{n}\left(\epsilon_{i} x_{i}+e_{i} y_{i}\right) \leq N\left(Z_{0}\right)\right\}$ is also a required compact and positively invariant set. The proof of this lemma is similar to Lemma 3.2, hence we omit it.

Positive equilibria existence can be obtained by the next formula.

$$
\left\{\begin{array}{l}
r_{i}-b_{i} x_{i}-e_{i} y_{i}+\sum_{j=1}^{n} d_{i j}^{x y} y_{j}=0, \\
-\gamma_{i}-\delta_{i} y_{i}+\varepsilon_{i} x_{i}+\sum_{j=1}^{n} d_{i j}^{y x} x_{j}=0, \quad i=1, \ldots, n .
\end{array}\right.
$$

Assume

$$
Z^{T}=\left(x_{1}, y_{1}, x_{2}, y_{2}, \ldots, x_{n}, y_{n}\right), \quad b^{T}=\left(r_{1},-\gamma_{1}, r_{2},-\gamma_{2}, \ldots, r_{n},-\gamma_{n}\right)
$$

Let

$$
A=\left(\begin{array}{cccccccc}
-b_{1} & -e_{1} & 0 & d_{12}^{x y} & 0 & d_{12}^{x y} & \ldots & \ldots \\
\varepsilon_{1} & -\delta_{1} & d_{12}^{y x} & 0 & d_{13}^{y x} & 0 & \ldots & \ldots \\
0 & d_{21}^{x y} & -b_{2} & -e_{2} & 0 & d_{23}^{x y} & \ldots & \ldots \\
d_{21}^{y x} & 0 & \varepsilon_{2} & -\delta_{2} & d_{23}^{y x} & 0 & \ldots & \ldots \\
\vdots & \vdots & \vdots & \vdots & \vdots & \vdots & \vdots \ldots & \vdots \ldots
\end{array}\right) .
$$


Consider the system of linear equations:

$$
A Z+b=0
$$

It is reasonable to require that the unique solution be positive. Therefore, positive equilibria for system (2) exist naturally. The next theorem is obtained as follows:

Theorem 3.1 If the unique solution exists and is positive for the system of linear equations $A Z+b=0$, the positive equilibrium for system (2) exists.

In fact, Cramer's rule can be used to prove that the unique solution exists and is positive for the system of linear equations $A Z+b=0$.

In this paper, we suppose conditions $\left(\mathbf{H}_{\mathbf{1}}\right)$ and $\left(\mathbf{H}_{\mathbf{2}}\right)$ are satisfied for model (2) as follows:

$\left(\mathbf{H}_{\mathbf{1}}\right) \frac{\left[\varepsilon_{i} b_{i}-\sum_{j=1}^{n}\left(\varepsilon_{i}\left|d_{i j}^{x y}\right|+e_{j}\left|d_{j i}^{y x}\right|\right)\right]}{\varepsilon_{i}^{2}}>0(i=1,2, \ldots, n)$.

$\left(\mathbf{H}_{2}\right) \frac{\left[e_{i} \delta_{i}-\sum_{j=1}^{n}\left(e_{i}\left|d_{i j}^{y x}\right|+\varepsilon_{j} \mid d_{j i}^{x y}\right)\right]}{e_{i}^{2}}>0(i=1,2, \ldots, n)$.

This means that the positive solution exists for model (2).

\subsection{Global-stability analysis for new model (2) based on strongly connected graphs}

Two matrices are constructed as follows:

$$
P=\left(p_{i j}\right)_{n \times n},
$$

with

$$
\begin{aligned}
& p_{i j}= \begin{cases}d_{i j}^{x y}, & i>j, \\
d_{i j}^{y x}, & i \leq j,\end{cases} \\
& F=\left(f_{i j}\right)_{n \times n},
\end{aligned}
$$

with

$$
f_{i j}= \begin{cases}d_{i j}^{y x}, & i>j, \\ d_{i j}^{x y}, & i \leq j .\end{cases}
$$

Let

$$
B=\left(\beta_{i j}\right)_{n \times n},
$$

where

$$
\beta_{i j}= \begin{cases}\varepsilon_{i} p_{i j}, & \left|\varepsilon_{i} p_{i j}\right| \geq\left|e_{i} f_{i j}\right|, i>j, \\ e_{i} f_{i j}, & \left|\varepsilon_{i} p_{i j}\right|<\left|e_{i} f_{i j}\right|, i>j, \\ e_{i} p_{i j}, & \left|e_{i} p_{i j}\right| \geq\left|\varepsilon_{i} f_{i j}\right|, i \leq j, \\ \varepsilon_{i} f_{i j}, & \left|e_{i} p_{i j}\right|<\left|\varepsilon_{i} f_{i j}\right|, i \leq j .\end{cases}
$$


A cross-dispersal matrix can be defined as follows:

$$
R=\left(\left|\beta_{i j}\right|\right)_{n \times n} .
$$

A digraph $(G, A)$ with $n$ vertices for system (2) can be constructed as follows. Each vertex represents a patch. At each vertex $i$ of $G$, vertex dynamics are described by the following system:

$$
\left\{\begin{array}{l}
\dot{x}_{i}=x_{i}\left(r_{i}-b_{i} x_{i}-e_{i} y_{i}\right), \\
\dot{y}_{i}=y_{i}\left(-\gamma_{i}-\delta_{i} y_{i}+\varepsilon_{i} x_{i}\right) .
\end{array}\right.
$$

Let $E(G)$ denote the set of arcs $(i, j)$ leading from initial vertex $i$ to terminal vertex $j$. We require that $(j, i) \in E(G)$ if and only if $d_{i j}^{x y} \neq 0$ or $d_{i j}^{y x} \neq 0$.

In this section, a predator-prey model with cross-dispersal is studied. By using the method of constructing Lyapunov functions based on a graph-theoretical approach for coupled systems, sufficient conditions that the positive equilibrium of coupling model (2) is globally asymptotically stable in $R_{+}^{2 n}$ are derived.

We obtain the main theorem as follows:

Theorem 3.2 Assume the following conditions hold:

1. Diagraph $(G, A)$ is balanced;

2. Cross-dispersal matrix $R=\left(\left|\beta_{i j}\right|\right)_{n \times n}$ is irreducible;

3. There exists a non-negative constant $\lambda$ such that

$$
-\lambda \varepsilon_{i} p_{i j}=e_{i} f_{i j} \quad(i>j), \quad-\lambda e_{i} p_{i j}=\varepsilon_{i} f_{i j} \quad(i \leq j) ;
$$

then, whenever a positive equilibrium $E^{*}=\left(x_{1}^{*}, y_{1}^{*}, x_{2}^{*}, y_{2}^{*}, \ldots, x_{n}^{*}, y_{n}^{*}\right)$ exists for system (2), it is unique and globally asymptotically stable in $R_{+}^{2 n}$.

Proof Let

$$
f_{i}\left(x_{i}, y_{i}\right)=r_{i}-b_{i} x_{i}-e_{i} y_{i}, \quad g_{i}\left(x_{i}, y_{i}\right)=-\gamma_{i}-\delta_{i} y_{i}+\varepsilon_{i} x_{i} .
$$

In the following, we have

$$
f_{i}\left(x_{i}^{*}, y_{i}^{*}\right)=-\sum_{j=1}^{n} d_{i j}^{x y} y_{j}^{*}, \quad g_{i}\left(x_{i}^{*}, y_{i}^{*}\right)=-\sum_{j=1}^{n} d_{i j}^{y x} x_{j}^{*} .
$$

Set the Lyapunov functions as

$$
V_{i}(t)=\varepsilon_{i}\left(x_{i}-x_{i}^{*}-x_{i}^{*} \ln \frac{x_{i}}{x_{i}^{*}}\right)+e_{i}\left(y_{i}-y_{i}-y_{i}^{*} \ln \frac{y_{i}}{y_{i}^{*}}\right) .
$$


Directly differentiating $V_{i}$ along system (2), we have

$$
\begin{aligned}
\dot{V}_{i}(t)= & \varepsilon_{i}\left(x_{i}-x_{i}^{*}\right)\left[f_{i}\left(x_{i}, y_{i}\right)-f_{i}\left(x_{i}^{*}, y_{i}^{*}\right)\right]+e_{i}\left(y_{i}-y_{i}^{*}\right)\left[g_{i}\left(x_{i}, y_{i}\right)-g_{i}\left(x_{i}^{*}, y_{i}^{*}\right)\right] \\
& +\sum_{j=1}^{n} d_{i j}^{x y} \varepsilon_{i}\left(x_{i}-x_{i}^{*}\right)\left(y_{j}-y_{j}^{*}\right)+\sum_{j=1}^{n} d_{i j}^{y x} e_{i}\left(y_{i}-y_{i}^{*}\right)\left(x_{j}-x_{j}^{*}\right) \\
= & -\varepsilon_{i} b_{i}\left(x_{i}-x_{i}^{*}\right)^{2}-e_{i} \delta_{i}\left(y_{i}-y_{i}^{*}\right)^{2} \\
& +\sum_{j=1}^{n} d_{i j}^{x y} \varepsilon_{i}\left(x_{i}-x_{i}^{*}\right)\left(y_{j}-y_{j}^{*}\right)+\sum_{j=1}^{n} d_{i j}^{y x} e_{i}\left(y_{i}-y_{i}^{*}\right)\left(x_{j}-x_{j}^{*}\right) .
\end{aligned}
$$

Two cases are discussed as follows:

Case I. $0 \leq \lambda \leq 1$.

\section{Choosing}

$$
a_{i j}=\left\{\begin{array}{ll}
\varepsilon_{i}\left|p_{i j}\right|, & i>j, \\
e_{i}\left|p_{i j}\right|, & i \leq j,
\end{array} \quad b_{i j}= \begin{cases}e_{i}\left|f_{i j}\right|, & i>j, \\
\varepsilon_{i}\left|f_{i j}\right|, & i \leq j .\end{cases}\right.
$$

Then, we obtain

$$
\left|\varepsilon_{i} p_{i j}\right| \geq\left|e_{i} f_{i j}\right| \quad(i>j)
$$

and

$$
\left|e_{i} p_{i j}\right| \geq\left|\varepsilon_{i} f_{i j}\right| \quad(i \leq j) .
$$

Therefore, the cross-dispersal matrix is obtained as follows:

$$
R=\left(\left|\beta_{i j}\right|\right)_{n \times n^{\prime}}
$$

where

$$
\beta_{i j}= \begin{cases}\varepsilon_{i} p_{i j}, & i>j, \\ e_{i} p_{i j}, & i \leq j .\end{cases}
$$

In the following, we have

$$
A=\left(a_{i j}\right)_{n \times n}=R=\left(\left|\beta_{i j}\right|\right)_{n \times n} .
$$

Let $c_{i}$ denote the cofactor of the $i$ th diagonal element of the matrix $\left(a_{i j}\right)_{n \times n}$. From the irreducible character of matrix $\left(a_{i j}\right)_{n \times n}$, we have $c_{i}>0$.

Furthermore, a Lyapunov function is set as follows:

$$
V(t)=\sum_{i=1}^{n} c_{i} V_{i}(t)
$$


Differentiating $V$ along the solution of system (2), we obtain

$$
\begin{aligned}
\dot{V}(t) \leq & -\sum_{i=1}^{n} c_{i} \varepsilon_{i} b_{i}\left(x_{i}-x_{i}^{*}\right)^{2}-\sum_{i=1}^{n} c_{i} e_{i} \delta_{i}\left(y_{i}-y_{i}^{*}\right)^{2} \\
& +\sum_{i, j=1}^{n} c_{i} a_{i j} F_{i j}(t)+\sum_{i, j=1}^{n} c_{i} b_{i j} F_{i j}^{2}(t) \\
\leq & -\sum_{i=1}^{n} c_{i} \varepsilon_{i} b_{i}\left(x_{i}-x_{i}^{*}\right)^{2}-\sum_{i=1}^{n} c_{i} e_{i} \delta_{i}\left(y_{i}-y_{i}^{*}\right)^{2} \\
& +\sum_{i, j=1}^{n} c_{i} a_{i j} F_{i j}(t)+\sum_{i, j=1}^{n} c_{i} \lambda a_{i j} F_{i j}^{2}(t),
\end{aligned}
$$

where

$$
\begin{gathered}
F_{i j}(t)= \begin{cases}\operatorname{sgn}\left(p_{i j}\right)\left(x_{i}-x_{i}^{*}\right)\left(y_{j}-y_{j}^{*}\right), & i>j, \\
\operatorname{sgn}\left(p_{i j}\right)\left(x_{j}-x_{j}^{*}\right)\left(y_{i}-y_{i}^{*}\right), & i \leq j,\end{cases} \\
F_{i j}^{2}(t)= \begin{cases}\operatorname{sgn}\left(f_{i j}\right)\left(x_{j}-x_{j}^{*}\right)\left(y_{i}-y_{i}^{*}\right), & i>j, \\
\operatorname{sgn}\left(f_{i j}\right)\left(x_{i}-x_{i}^{*}\right)\left(y_{j}-y_{j}^{*}\right), & i \leq j,\end{cases}
\end{gathered}
$$

Furthermore, we obtain that

$$
\operatorname{sgn}\left(p_{i j}\right)=-\operatorname{sgn}\left(p_{j i}\right), \quad \operatorname{sgn}\left(f_{i j}\right)=-\operatorname{sgn}\left(f_{j i}\right) .
$$

Because the cross-dispersal matrix $R=\left(\left|\beta_{i j}\right|\right)_{n \times n}$ is irreducible, the diagraph $(G, A)$ is strongly connected. Furthermore, since diagraph $(G, A)$ is balanced and strongly connected, we obtain that

$$
\begin{aligned}
& \sum_{i=1}^{n} c_{i} a_{i j} F_{i j}(t) \\
&=\frac{1}{2} \sum_{Q \in \mathrm{Q}} w(Q) \sum_{(j, i) \in E\left(C_{\mathrm{Q}}\right)}\left[F_{i j}(t)+F_{j i}(t)\right] \\
&=\frac{1}{2} \sum_{Q \in \mathrm{Q}} w(Q) \sum_{(j, i) \in E\left(C_{\mathrm{Q}}\right)(i>j)}\left[\operatorname{sgn}\left(p_{i j}\right)\left(y_{j}-y_{j}^{*}\right)\left(x_{i}-x_{i}^{*}\right)+\operatorname{sgn}\left(p_{j i}\right)\left(x_{i}-x_{i}^{*}\right)\left(y_{j}-y_{j}^{*}\right)\right] \\
&+\frac{1}{2} \sum_{Q \in \mathrm{Q}} w(Q) \sum_{(j, i) \in E\left(C_{\mathrm{Q}}\right)(i \leq j)}\left[\operatorname{sgn}\left(p_{i j}\right)\left(y_{i}-y_{i}^{*}\right)\left(x_{j}-x_{j}^{*}\right)+\operatorname{sgn}\left(p_{j i}\right)\left(y_{i}-y_{i}^{*}\right)\left(x_{j}-x_{j}^{*}\right)\right] \\
&=\frac{1}{2} \sum_{Q \in \mathrm{Q}} w(Q) \sum_{(j, i) \in E\left(C_{\mathrm{Q}}\right)(i>j)}\left[\operatorname{sgn}\left(p_{i j}\right)\left(y_{j}-y_{j}^{*}\right)\left(x_{i}-x_{i}^{*}\right)-\operatorname{sgn}\left(p_{i j}\right)\left(x_{i}-x_{i}^{*}\right)\left(y_{j}-y_{j}^{*}\right)\right] \\
&+\frac{1}{2} \sum_{Q \in \mathrm{Q}} w(Q) \sum_{(j, i) \in E\left(C_{\mathrm{Q}}\right)(i \leq j)}\left[\operatorname{sgn}\left(p_{i j}\right)\left(y_{i}-y_{i}^{*}\right)\left(x_{j}-x_{j}^{*}\right)-\operatorname{sgn}\left(p_{i j}\right)\left(y_{i}-y_{i}^{*}\right)\left(x_{j}-x_{j}^{*}\right)\right] \\
&= 0+0 \\
&= 0,
\end{aligned}
$$




$$
\begin{aligned}
\sum_{i=1}^{n} c_{i} \lambda a_{i j} F_{i j}^{2}(t) & \\
= & \frac{\lambda}{2} \sum_{Q \in \mathrm{Q}} w(Q) \sum_{(j, i) \in E\left(C_{Q}\right)}\left[F_{i j}^{2}(t)+F_{j i}^{2}(t)\right] \\
= & \frac{\lambda}{2} \sum_{Q \in \mathrm{Q}} w(Q) \sum_{(j, i) \in E\left(C_{Q}\right)(i>j)}\left[\operatorname{sgn}\left(f_{i j}\right)\left(y_{i}-y_{i}^{*}\right)\left(x_{j}-x_{j}^{*}\right)+\operatorname{sgn}\left(f_{j i}\right)\left(x_{j}-x_{j}^{*}\right)\left(y_{i}-y_{i}^{*}\right)\right] \\
& +\frac{\lambda}{2} \sum_{Q \in \mathrm{Q}} w(Q) \sum_{(j, i) \in E\left(C_{Q}\right)(i \leq j)}\left[\operatorname{sgn}\left(f_{i j}\right)\left(y_{j}-y_{j}^{*}\right)\left(x_{i}-x_{i}^{*}\right)+\operatorname{sgn}\left(f_{j i}\right)\left(y_{j}-y_{j}^{*}\right)\left(x_{i}-x_{i}^{*}\right)\right] \\
= & \frac{\lambda}{2} \sum_{Q \in \mathrm{Q}} w(Q) \sum_{(j, i) \in E\left(C_{Q}\right)(i>j)}\left[\operatorname{sgn}\left(f_{i j}\right)\left(y_{i}-y_{i}^{*}\right)\left(x_{j}-x_{j}^{*}\right)-\operatorname{sgn}\left(f_{i j}\right)\left(x_{j}-x_{j}^{*}\right)\left(y_{i}-y_{i}^{*}\right)\right] \\
& +\frac{\lambda}{2} \sum_{Q \in \mathrm{Q}} w(Q) \sum_{(j, i) \in E\left(C_{Q}\right)(i \leq j)}\left[\operatorname{sgn}\left(f_{i j}\right)\left(y_{j}-y_{j}^{*}\right)\left(x_{i}-x_{i}^{*}\right)-\operatorname{sgn}\left(f_{i j}\right)\left(y_{j}-y_{j}^{*}\right)\left(x_{i}-x_{i}^{*}\right)\right] \\
= & 0+0 \\
= & 0 .
\end{aligned}
$$

In addition, we have

$$
\dot{V}(t) \leq-\sum_{i=1}^{n} c_{i} \varepsilon_{i} b_{i}\left(x_{i}-x_{i}^{*}\right)^{2}-\sum_{i=1}^{n} c_{i} e_{i} \delta_{i}\left(y_{i}-y_{i}^{*}\right)^{2}
$$

Therefore, by the LaSalle Invariance Principle [2], $E^{*}$ is unique and globally asymptotically stable in $R_{+}^{2 n}$.

Case II. $\lambda>1$.

In this case, the cross-dispersal matrix

$$
R=\left(\left|\beta_{i j}\right|\right)_{n \times n}=\left(b_{i j}\right)_{n \times n} .
$$

Let $c_{i}$ denote the cofactor of the $i$ th diagonal element of the matrix $\left(b_{i j}\right)_{n \times n}$. From the irreducible character of matrix $\left(b_{i j}\right)_{n \times n}$, we have $c_{i}>0$.

Furthermore, a Lyapunov function is listed as follows:

$$
V(t)=\sum_{i=1}^{n} c_{i} V_{i}(t)
$$

After calculation, we obtain

$$
\begin{aligned}
\dot{V}(t) \leq & -\sum_{i=1}^{n} c_{i} \varepsilon_{i} b_{i}\left(x_{i}-x_{i}^{*}\right)^{2}-\sum_{i=1}^{n} c_{i} e_{i} \delta_{i}\left(y_{i}-y_{i}^{*}\right)^{2} \\
& +\sum_{i, j=1}^{n} c_{i} \lambda b_{i j} F_{i j}(t)+\sum_{i, j=1}^{n} c_{i} b_{i j} F_{i j}^{2}(t) .
\end{aligned}
$$


Similar to Case I, we obtain

$$
\sum_{i, j=1}^{n} c_{i} \lambda b_{i j} F_{i j}(t)=0, \quad \sum_{i, j=1}^{n} c_{i} b_{i j} F_{i j}^{2}(t)=0 .
$$

Hence,

$$
\dot{V}(t) \leq-\sum_{i=1}^{n} c_{i} \varepsilon_{i} b_{i}\left(x_{i}-x_{i}^{*}\right)^{2}-\sum_{i=1}^{n} c_{i} e_{i} \delta_{i}\left(y_{i}-y_{i}^{*}\right)^{2}
$$

Therefore, by the LaSalle Invariance Principle [2], $E^{*}$ is unique and globally asymptotically stable in $R_{+}^{2 n}$.

From Case I and Case II, the proof is completed.

Consider $\lambda=0$ about Theorem 3.2, we have the following corollary:

Corollary 3.1 Assume that the following assumptions hold for system (2):

1. Diagraph $(G, A)$ is balanced;

2. Cross-dispersal matrix $R=\left(\left|\beta_{i j}\right|\right)_{n \times n}$ is irreducible;

3. $d_{i j}^{y x}=0(i>j), d_{i j}^{x y}=0(i \leq j)$;

then, whenever a positive equilibrium $E^{*}=\left(x_{1}^{*}, y_{1}^{*}, x_{2}^{*}, y_{2}^{*}, \ldots, x_{n}^{*}, y_{n}^{*}\right)$ exists, it is unique and globally asymptotically stable in $R_{+}^{2 n}$.

If the condition 3 of Theorem 3.2 is substituted for the formula as follows:

$$
-\varepsilon_{i} p_{i j}=\lambda e_{i} f_{i j} \quad(i>j), \quad-e_{i} p_{i j}=\lambda \varepsilon_{i} f_{i j} \quad(i \leq j),
$$

then, we have the following corollary:

Corollary 3.2 Assume the following conditions hold:

1. Diagraph $(G, A)$ is balanced;

2. Cross-dispersal matrix $R=\left(\left|\beta_{i j}\right|\right)_{n \times n}$ is irreducible;

3. There exists a non-negative constant $\lambda$ such that

$$
-\varepsilon_{i} p_{i j}=\lambda e_{i} f_{i j} \quad(i>j), \quad-e_{i} p_{i j}=\lambda \varepsilon_{i} f_{i j} \quad(i \leq j)
$$

then, whenever a positive equilibrium $E^{*}=\left(x_{1}^{*}, y_{1}^{*}, x_{2}^{*}, y_{2}^{*}, \ldots, x_{n}^{*}, y_{n}^{*}\right)$ exists, it is unique and globally asymptotically stable in $R_{+}^{2 n}$.

Consider $\lambda=0$ about Corollary 3.2, we have the following corollary:

Corollary 3.3 Assume that the following assumptions hold for system (2):

1. Diagraph $(G, A)$ is balanced;

2. Cross-dispersal matrix $R=\left(\left|\beta_{i j}\right|\right)_{n \times n}$ is irreducible;3. $d_{i j}^{x y}=0(i>j), d_{i j}^{y x}=0(i \leq j)$; then, whenever a positive equilibrium $E^{*}=\left(x_{1}^{*}, y_{1}^{*}, x_{2}^{*}, y_{2}^{*}, \ldots, x_{n}^{*}, y_{n}^{*}\right)$ exists, it is unique and globally asymptotically stable in $R_{+}^{2 n}$. 


\subsection{Global-stability analysis for new model (2) based on strongly connected components}

Let $\left(R_{h k}, B_{h k}\right)$ denote the $k$ th strongly connected component (SCC) of the $h$ th layer of a network $(G, A) . V\left(R_{h k}\right)$ denotes the vertex set of the SCC $\left(R_{h k}, B_{h k}\right)$ and $N_{h k}$ denotes the number of vertices of the $\operatorname{SCC}\left(R_{h k}, B_{h k}\right)$.

Obviously,

$$
\sum \sum N_{h k}=n
$$

Then system (2) can be written as follows:

When $h=1$, system (2) is restricted on the first layer of $(G, A)$, i.e.

$$
\left\{\begin{array}{l}
\dot{x}_{i}=x_{i}\left(r_{i}-b_{i} x_{i}-e_{i} y_{i}\right)+\sum_{j \in V\left(R_{1 k}\right)} d_{i j}^{x y} y_{j} x_{i}, \\
\dot{y}_{i}=y_{i}\left(-\gamma_{i}-\delta_{i} y_{i}+\varepsilon_{i} x_{i}\right)+\sum_{j \in V\left(R_{1 k}\right)} d_{i j}^{y x} x_{j} y_{i} \quad\left(i \in V\left(R_{1 k}\right)\right) .
\end{array}\right.
$$

When $h>1$, system (2) is restricted on the $h$ th layer of $(G, A)$, i.e.

$$
\left\{\begin{aligned}
\dot{x}_{i}= & x_{i}\left(r_{i}-b_{i} x_{i}-e_{i} y_{i}\right)+\sum_{j \in \cup_{1 \leq l<h} \cup_{m} V\left(R_{l m}\right)} d_{i j}^{x y} y_{j} x_{i}+\sum_{j \in V\left(R_{h k}\right)} d_{i j}^{x y} y_{j} x_{i} \\
\dot{y}_{i}= & y_{i}\left(-\gamma_{i}-\delta_{i} y_{i}+\varepsilon_{i} x_{i}\right)+\sum_{j \in \cup_{1 \leq l<h} \bigcup_{m} V\left(R_{l m}\right)} d_{i j}^{y x} x_{j} y_{i} \\
& +\sum_{j \in V\left(R_{h k}\right)} d_{i j}^{y x} x_{j} y_{i} \quad\left(i \in V\left(R_{h k}\right)\right) .
\end{aligned}\right.
$$

Based on the theory of asymptotically autonomous systems, graph theory and Lyapunov theory, a global-stability theorem without strongly connected graphs is established in this section.

The main theorem is obtained as follows:

Theorem 3.3 Assume the following conditions hold:

1. Diagraph $(G, A)$ is balanced;

2. Cross-dispersal matrix $R=\left(\left|\beta_{i j}\right|\right)_{n \times n}$ is reducible. (This means diagraph $(G, A)$ is not strongly connected.);

3. $\left(R_{h k}, B_{h k}\right)$ are strongly connected components $(S C C)$ of diagraph $(G, A)$;

4. There exists a non-negative constant $\lambda$ such that

$$
-\lambda \varepsilon_{i} p_{i j}=e_{i} f_{i j} \quad(i>j), \quad-\lambda e_{i} p_{i j}=\varepsilon_{i} f_{i j} \quad(i \leq j) ;
$$

then, whenever a positive equilibrium $E^{*}=\left(x_{1}^{*}, y_{1}^{*}, x_{2}^{*}, y_{2}^{*}, \ldots, x_{n}^{*}, y_{n}^{*}\right)$ exists for system (2), it is unique and globally asymptotically stable in $R_{+}^{2 n}$.

Proof Step 1 . Consider the strongly connected component $\left(R_{1 k}, B_{1 k}\right)$. The next system is obtained naturally.

$$
\left\{\begin{array}{l}
\dot{x}_{i}=x_{i}\left(r_{i}-b_{i} x_{i}-e_{i} y_{i}\right)+\sum_{j \in V\left(R_{1 k}\right)} d_{i j}^{x y} y_{j} x_{i}, \\
\dot{y}_{i}=y_{i}\left(-\gamma_{i}-\delta_{i} y_{i}+\varepsilon_{i} x_{i}\right)+\sum_{j \in V\left(R_{1 k}\right)} d_{i j}^{y x} x_{j} y_{i} \quad\left(i \in V\left(R_{1 k}\right)\right) .
\end{array}\right.
$$


A vertex Lyapunov function on the $\operatorname{SCC}\left(R_{1 k}, B_{1 k}\right)$ is constructed as follows:

$$
V_{1 k}(t)=\sum_{i \in V\left(R_{1 k}\right)} c_{i}^{1 k} V_{i}(t)
$$

where

$$
V_{i}(t)=\varepsilon_{i}\left(x_{i}-x_{i}^{*}-x_{i}^{*} \ln \frac{x_{i}}{x_{i}^{*}}\right)+e_{i}\left(y_{i}-y_{i}^{*}-y_{i}^{*} \ln \frac{y_{i}}{y_{i}^{*}}\right) .
$$

Let $c_{i}^{1 k}$ denote the cofactor of the $k$ th diagonal element of the matrix $L_{1 k}$. Here, $L_{1 k}$ is the $\operatorname{SCC}\left(R_{1 k}, B_{1 k}\right)$ 's Laplacian Matrix. As $\left(R_{1 k}, B_{1 k}\right)$ is strongly connected, we obtain $c_{i}^{1 k}>0$ for every $i \in V\left(R_{1 k}\right)$.

Let us assume $0 \leq \lambda \leq 1$. Choosing

$$
a_{i j}^{1 k}=\left\{\begin{array}{ll}
\varepsilon_{i}\left|p_{i j}\right|, & i>j, \\
e_{i}\left|p_{i j}\right|, & i \leq j,
\end{array} \quad b_{i j}^{1 k}= \begin{cases}e_{i}\left|f_{i j}\right|, & i>j, \\
\varepsilon_{i}\left|f_{i j}\right|, & i \leq j .\end{cases}\right.
$$

Similar to Theorem 3.2, we obtain

$$
\begin{aligned}
\dot{V}_{1 k}(t) \leq & -\sum_{i \in V\left(R_{1 k}\right)} c_{i}^{1 k} \varepsilon_{i} b_{i}\left(x_{i}-x_{i}^{*}\right)^{2}-\sum_{i \in V\left(R_{1 k}\right)} c_{i}^{1 k} e_{i} \delta_{i}\left(y_{i}-y_{i}^{*}\right)^{2} \\
& +\sum_{i, j \in V\left(R_{1 k}\right)} c_{i}^{1 k} a_{i j}^{1 k} F_{i j}(t)+\sum_{i, j \in V\left(R_{1 k}\right)} c_{i}^{1 k} b_{i j}^{1 k} F_{i j}^{2}(t) \\
\leq & -\sum_{i \in V\left(R_{1 k}\right)} c_{i}^{1 k} \varepsilon_{i} b_{i}\left(x_{i}-x_{i}^{*}\right)^{2}-\sum_{i \in V\left(R_{1 k}\right)} c_{i}^{1 k} e_{i} \delta_{i}\left(y_{i}-y_{i}^{*}\right)^{2} \\
& +\sum_{i, j \in V\left(R_{1 k}\right)} c_{i}^{1 k} a_{i j}^{1 k} F_{i j}(t)+\sum_{i, j \in V\left(R_{1 k}\right)} c_{i}^{1 k} \lambda a_{i j}^{1 k} F_{i j}^{2}(t) .
\end{aligned}
$$

Since diagraph $(G, A)$ is balanced, diagraph $\left(R_{1 k}, B_{1 k}\right)$ is considered to be balanced naturally. As $\left(a_{i j}^{1 k}\right)_{N_{1 k} \times N_{1 k}}$ is irreducible and diagraph $\left(R_{1 k}, B_{1 k}\right)$ is balanced, we obtain

$$
\sum_{i, j \in V\left(R_{1 k}\right)} c_{i}^{1 k} a_{i j}^{1 k} F_{i j}(t)=0, \quad \sum_{i, j \in V\left(R_{1 k}\right)} c_{i}^{1 k} \lambda a_{i j}^{1 k} F_{i j}^{2}(t)=0 .
$$

Hence,

$$
\dot{V}_{1 k}(t) \leq-\sum_{i \in V\left(R_{1 k}\right)} c_{i}^{1 k} \varepsilon_{i} b_{i}\left(x_{i}-x_{i}^{*}\right)^{2}-\sum_{i \in V\left(R_{1 k}\right)} c_{i}^{1 k} e_{i} \delta_{i}\left(y_{i}-y_{i}^{*}\right)^{2} .
$$

If $\lambda>1$, the proof is similar. Hence, system (2) is globally asymptotically stable on the SCC $\left(R_{1 k}, B_{1 k}\right)$.

Step 2 . Consider the strongly connected component $\left(R_{2 k}, B_{2 k}\right)$, we obtain

$$
\left\{\begin{array}{l}
\dot{x}_{i}=x_{i}\left(r_{i}-b_{i} x_{i}-e_{i} y_{i}\right)+\sum_{j \in V\left(R_{1 k}\right)} d_{i j}^{x y} y_{j} x_{i}+\sum_{j \in V\left(R_{2 k}\right)} d_{i j}^{x y} y_{j} x_{i}, \\
\dot{y}_{i}=y_{i}\left(-\gamma_{i}-\delta_{i} y_{i}+\varepsilon_{i} x_{i}\right)+\sum_{j \in V\left(R_{1 k}\right)} d_{i j}^{y x} x_{j} y_{i}+\sum_{j \in V\left(R_{2 k}\right)} d_{i j}^{y x} x_{j} y_{i} \quad\left(i \in V\left(R_{2 k}\right)\right) .
\end{array}\right.
$$


According to the theory of asymptotically autonomous systems, we have

$$
\left\{\begin{array}{l}
\dot{x}_{i}=x_{i}\left(r_{i}-b_{i} x_{i}-e_{i} y_{i}\right)+\sum_{j \in V\left(R_{1 k}\right)} d_{i j}^{x y} y_{j}^{*} x_{i}+\sum_{j \in V\left(R_{2 k}\right)} d_{i j}^{x y} y_{j} x_{i}, \\
\dot{y}_{i}=y_{i}\left(-\gamma_{i}-\delta_{i} y_{i}+\varepsilon_{i} x_{i}\right)+\sum_{j \in V\left(R_{1 k}\right)} d_{i j}^{y x} x_{j}^{*} y_{i}+\sum_{j \in V\left(R_{2 k}\right)} d_{i j}^{y x} x_{j} y_{i} \quad\left(i \in V\left(R_{2 k}\right)\right) .
\end{array}\right.
$$

Similarly, a Lyapunov function can be constructed as follows:

$$
V_{2 k}(t)=\sum_{i \in V\left(R_{2 k}\right)} c_{i}^{2 k} V_{i}(t)
$$

where

$$
V_{i}(t)=\varepsilon_{i}\left(x_{i}-x_{i}^{*}-x_{i}^{*} \ln \frac{x_{i}}{x_{i}^{*}}\right)+e_{i}\left(y_{i}-y_{i}-y_{i}^{*} \ln \frac{y_{i}}{y_{i}^{*}}\right) .
$$

Let $c_{i}^{2 k}$ denote the cofactor of the $k$ th diagonal element of the matrix $L_{2 k}$. Here, $L_{2 k}$ is the SCC $\left(R_{2 k}, B_{2 k}\right)$ 's Laplacian Matrix. As $\left(R_{2 k}, B_{2 k}\right)$ is strongly connected, we obtain $c_{i}^{2 k}>0$ for every $i \in V\left(R_{2 k}\right)$.

Let us assume $\lambda>1$. Choosing

$$
a_{i j}^{2 k}=\left\{\begin{array}{ll}
\varepsilon_{i}\left|p_{i j}\right|, & i>j, \\
e_{i}\left|p_{i j}\right|, & i \leq j,
\end{array} \quad b_{i j}^{2 k}= \begin{cases}e_{i}\left|f_{i j}\right|, & i>j, \\
\varepsilon_{i}\left|f_{i j}\right|, & i \leq j .\end{cases}\right.
$$

Similar to Step 1, we obtain that

$$
\begin{aligned}
\dot{V}_{2 k}(t) \leq & -\sum_{i \in V\left(R_{2 k}\right)} c_{i}^{2 k} \varepsilon_{i} b_{i}\left(x_{i}-x_{i}^{*}\right)^{2}-\sum_{i \in V\left(R_{2 k}\right)} c_{i}^{2 k} e_{i} \delta_{i}\left(y_{i}-y_{i}^{*}\right)^{2} \\
& +\sum_{i, j \in V\left(R_{2 k}\right)} c_{i}^{2 k} a_{i j}^{2 k} F_{i j}(t)+\sum_{i, j \in V\left(R_{2 k}\right)} c_{i}^{2 k} b_{i j}^{2 k} F_{i j}^{2}(t) \\
\leq & -\sum_{i \in V\left(R_{2 k}\right)} c_{i}^{2 k} \varepsilon_{i} b_{i}\left(x_{i}-x_{i}^{*}\right)^{2}-\sum_{i \in V\left(R_{2 k}\right)} c_{i}^{2 k} e_{i} \delta_{i}\left(y_{i}-y_{i}^{*}\right)^{2} \\
& +\sum_{i, j \in V\left(R_{2 k}\right)} c_{i}^{2 k} \lambda b_{i j}^{2 k} F_{i j}(t)+\sum_{i, j \in V\left(R_{2 k}\right)} c_{i}^{2 k} b_{i j}^{2 k} F_{i j}^{2}(t) .
\end{aligned}
$$

Since diagraph $(G, A)$ is balanced, diagraph $\left(R_{2 k}, B_{2 k}\right)$ is considered to be balanced naturally. As $\left(b_{i j}^{2 k}\right)_{N_{2 k} \times N_{2 k}}$ is irreducible and diagraph $\left(R_{2 k}, B_{2 k}\right)$ is balanced, we obtain

$$
\sum_{i, j \in V\left(R_{2 k}\right)} c_{i}^{2 k} \lambda b_{i j}^{2 k} F_{i j}(t)=0, \quad \sum_{i, j \in V\left(R_{2 k}\right)} c_{i}^{2 k} b_{i j}^{2 k} F_{i j}^{2}(t)=0 .
$$

Hence,

$$
\dot{V}_{2 k}(t) \leq-\sum_{i \in V\left(R_{2 k}\right)} c_{i}^{2 k} \varepsilon_{i} b_{i}\left(x_{i}-x_{i}^{*}\right)^{2}-\sum_{i \in V\left(R_{2 k}\right)} c_{i}^{2 k} e_{i} \delta_{i}\left(y_{i}-y_{i}^{*}\right)^{2} .
$$

If $0 \leq \lambda \leq 1$, the proof is similar. Hence, system (2) is globally asymptotically stable on the $\operatorname{SCC}\left(R_{2 k}, B_{2 k}\right)$. 
Step 3. After repeating the above procedure for any $h k$, we obtain that system (2) is globally asymptotically stable on any $\mathrm{SCC}\left(R_{h k}, B_{h k}\right)$. A Lyapunov function is listed as follows:

$$
V(t)=\sum_{h, k} V_{h k}(t)
$$

Then, we obtain that

$$
\dot{V}(t) \leq-\sum_{h, k} \sum_{i \in V\left(R_{h k}\right)} c_{i}^{h k} \varepsilon_{i} b_{i}\left(x_{i}-x_{i}^{*}\right)^{2}-\sum_{h, k} \sum_{i \in V\left(R_{h k}\right)} c_{i}^{h k} e_{i} \delta_{i}\left(y_{i}-y_{i}^{*}\right)^{2} .
$$

Therefore, by the LaSalle Invariance Principle [2], $E^{*}$ is unique and globally asymptotically stable in $R_{+}^{2 n}$. In the following, the proof is completed.

The next corollaries are obtained naturally.

Corollary 3.4 Assume the following conditions hold for system (2):

1. Conditions $1-3$ of Theorem 3.3 are satisfied;

2. $d_{i j}^{y x}=0(i>j), d_{i j}^{x y}=0(i \leq j)$;

then, whenever a positive equilibrium $E^{*}=\left(x_{1}^{*}, y_{1}^{*}, x_{2}^{*}, y_{2}^{*}, \ldots, x_{n}^{*}, y_{n}^{*}\right)$ exists, it is unique and globally asymptotically stable in $R_{+}^{2 n}$.

Corollary 3.5 Assume the following conditions hold:

1. Conditions $1-3$ of Theorem 3.3 are satisfied;

2. There exists a non-negative constant $\lambda$ such that

$$
-\varepsilon_{i} p_{i j}=\lambda e_{i} f_{i j} \quad(i>j), \quad-e_{i} p_{i j}=\lambda \varepsilon_{i} f_{i j} \quad(i \leq j)
$$

then, whenever a positive equilibrium $E^{*}=\left(x_{1}^{*}, y_{1}^{*}, x_{2}^{*}, y_{2}^{*}, \ldots, x_{n}^{*}, y_{n}^{*}\right)$ exists for system $(2)$, it is unique and globally asymptotically stable in $R_{+}^{2 n}$.

Corollary 3.6 Assume the following conditions hold for system (2):

1. Conditions $1-3$ of Theorem 3.3 are satisfied;

2. $d_{i j}^{x y}=0(i>j), d_{i j}^{y x}=0(i \leq j)$;

then, whenever a positive equilibrium $E^{*}=\left(x_{1}^{*}, y_{1}^{*}, x_{2}^{*}, y_{2}^{*}, \ldots, x_{n}^{*}, y_{n}^{*}\right)$ exists, it is unique and globally asymptotically stable in $R_{+}^{2 n}$.

\section{Examples}

Example 4.1 An example is presented to illustrate Theorem 3.2. Consider the following predator-prey system with cross-dispersal:

$$
\left\{\begin{array}{l}
\dot{x}_{i}=x_{i}\left(r_{i}-b_{i} x_{i}-e_{i} y_{i}\right)+\sum_{j=1}^{n} d_{i j}^{x y} y_{j} x_{i}, \\
\dot{y}_{i}=y_{i}\left(-\gamma_{i}-\delta_{i} y_{i}+\varepsilon_{i} x_{i}\right)+\sum_{j=1}^{n} d_{i j}^{y x} x_{j} y_{i}, \quad i=1, \ldots, 8 .
\end{array}\right.
$$

The parameters are listed as follows.

$$
r_{1}=1, \quad b_{1}=0.5, \quad e_{1}=0.5, \quad \gamma_{1}=0.01, \quad \delta_{1}=0.5, \quad \varepsilon_{1}=0.5,
$$




$$
\begin{aligned}
& r_{2}=1.01, \quad b_{2}=0.5, \quad e_{2}=0.5, \quad \gamma_{2}=0.01, \quad \delta_{2}=0.5, \quad \varepsilon_{2}=0.5, \\
& r_{3}=1.01, \quad b_{3}=0.5, \quad e_{3}=0.5, \quad \gamma_{3}=0.02, \quad \delta_{3}=0.5, \quad \varepsilon_{3}=0.5 \text {, } \\
& r_{4}=1.02, \quad b_{4}=0.5, \quad e_{4}=0.5, \quad \gamma_{4}=0.01, \quad \delta_{4}=0.49, \quad \varepsilon_{4}=0.5 \text {, } \\
& r_{5}=1.01, \quad b_{5}=0.5, \quad e_{5}=0.5, \quad \gamma_{5}=0.02, \quad \delta_{5}=0.5, \quad \varepsilon_{5}=0.5 \text {, } \\
& r_{6}=1.01, \quad b_{6}=0.5, \quad e_{6}=0.5, \quad \gamma_{6}=0.01, \quad \delta_{6}=0.5, \quad \varepsilon_{6}=0.5 \text {, } \\
& r_{7}=1.01, \quad b_{7}=0.5, \quad e_{7}=0.5, \quad \gamma_{7}=0.01, \quad \delta_{7}=0.5, \quad \varepsilon_{7}=0.5 \text {, } \\
& r_{8}=1.02, \quad b_{8}=0.5, \quad e_{8}=0.5, \quad \gamma_{8}=0.01, \quad \delta_{8}=0.49, \quad \varepsilon_{8}=0.5 \text {. }
\end{aligned}
$$

Assume

$$
\begin{aligned}
& d_{12}^{y x}=d_{14}^{y x}=d_{23}^{y x}=d_{67}^{y x}=d_{78}^{y x}=d_{34}^{y x}=d_{35}^{y x}=d_{56}^{y x}=d_{58}^{y x}=0.01, \\
& d_{21}^{x y}=d_{32}^{x y}=d_{41}^{x y}=d_{43}^{x y}=d_{53}^{x y}=d_{65}^{x y}=d_{76}^{x y}=d_{85}^{x y}=d_{87}^{x y}=-0.01 .
\end{aligned}
$$

Otherwise,

$$
d_{i j}^{x y}=d_{i j}^{y x}=0 .
$$

Then, we can obtain that

$$
d_{i j}^{y x}=0 \quad(i>j), \quad d_{i j}^{x y}=0 \quad(i \leq j) .
$$

The cross-dispersal matrix is listed as follows:

$$
\begin{aligned}
R & =\left(\left|\beta_{i j}\right|\right)_{n \times n} \\
& =\left(\begin{array}{cccccccc}
0 & e_{1}\left|d_{12}^{y x}\right| & 0 & e_{1}\left|d_{14}^{y x}\right| & 0 & 0 & 0 & 0 \\
\varepsilon_{2}\left|d_{21}^{x y}\right| & 0 & e_{2}\left|d_{23}^{y x}\right| & 0 & 0 & 0 & 0 & 0 \\
0 & \varepsilon_{3}\left|d_{32}^{x y}\right| & 0 & e_{3}\left|d_{34}^{y x}\right| & e_{3}\left|d_{35}^{y x}\right| & 0 & 0 & 0 \\
\varepsilon_{4}\left|d_{41}^{x y}\right| & 0 & \varepsilon_{4}\left|d_{43}^{x y}\right| & 0 & 0 & 0 & 0 & 0 \\
0 & 0 & \varepsilon_{5}\left|d_{53}^{x y}\right| & 0 & 0 & e_{5}\left|d_{56}^{y x}\right| & 0 & e_{5}\left|d_{58}^{y x}\right| \\
0 & 0 & 0 & 0 & \varepsilon_{6}\left|d_{65}^{x y}\right| & 0 & e_{6}\left|d_{67}^{y x}\right| & 0 \\
0 & 0 & 0 & 0 & 0 & \varepsilon_{7}\left|d_{76}^{x y}\right| & 0 & e_{7}\left|d_{78}^{y x}\right| \\
0 & 0 & 0 & 0 & \varepsilon_{8}\left|d_{85}^{x y}\right| & 0 & \varepsilon_{8}\left|d_{87}^{x y}\right| & 0
\end{array}\right) .
\end{aligned}
$$

Simple computation results in

$$
R=\left(\begin{array}{cccccccc}
0 & 0.005 & 0 & 0.005 & 0 & 0 & 0 & 0 \\
0.005 & 0 & 0.005 & 0 & 0 & 0 & 0 & 0 \\
0 & 0.005 & 0 & 0.005 & 0.005 & 0 & 0 & 0 \\
0.005 & 0 & 0.005 & 0 & 0 & 0 & 0 & 0 \\
0 & 0 & 0.005 & 0 & 0 & 0.005 & 0 & 0.005 \\
0 & 0 & 0 & 0 & 0.005 & 0 & 0.005 & 0 \\
0 & 0 & 0 & 0 & 0 & 0.005 & 0 & 0.005 \\
0 & 0 & 0 & 0 & 0.005 & 0 & 0.005 & 0
\end{array}\right) .
$$




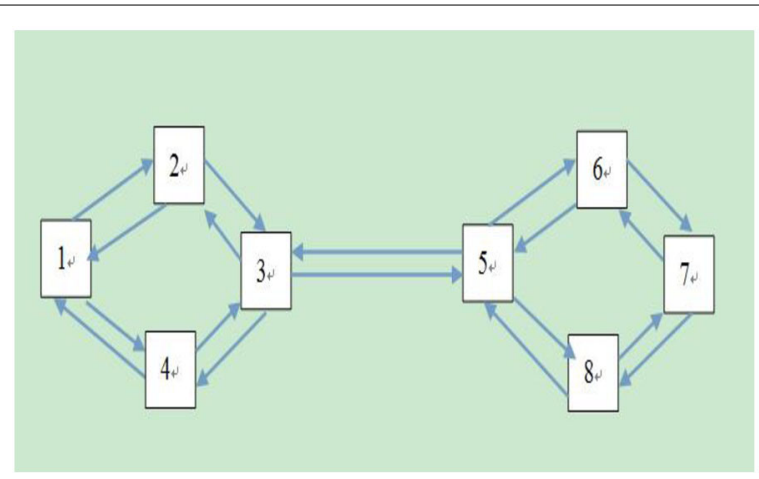

Figure 1 Strongly connected graph

The positive equilibrium for system (11) is obtained as follows:

$$
E^{*}=(1,1,1,1, \ldots, 1,1)
$$

From the construction of the graph, the relationship between vertices is shown in Fig. 1.

It is obvious that diagraph $(G, A)$ is strong connected and balanced. Using Corollary 3.1, we obtain that $E^{*}=(1,1,1,1, \ldots, 1,1)$ is unique and globally asymptotically stable.

Example 4.2 An example is presented to illustrate Theorem 3.3. Consider the following predator-prey system with cross-dispersal:

$$
\left\{\begin{array}{l}
\dot{x_{i}}=x_{i}\left(r_{i}-b_{i} x_{i}-e_{i} y_{i}\right)+\sum_{j=1}^{n} d_{i j}^{x y} y_{j} x_{i}, \\
\dot{y}_{i}=y_{i}\left(-\gamma_{i}-\delta_{i} y_{i}+\varepsilon_{i} x_{i}\right)+\sum_{j=1}^{n} d_{i j}^{y x} x_{j} y_{i}, \quad i=1, \ldots, 8 .
\end{array}\right.
$$

The parameters are listed as follows.

$$
\begin{aligned}
& r_{1}=1, \quad b_{1}=0.5, \quad e_{1}=0.5, \quad \gamma_{1}=0.01, \quad \delta_{1}=0.5, \quad \varepsilon_{1}=0.5, \\
& r_{2}=1.01, \quad b_{2}=0.5, \quad e_{2}=0.5, \quad \gamma_{2}=0.01, \quad \delta_{2}=0.5, \quad \varepsilon_{2}=0.5 \text {, } \\
& r_{3}=1.01, \quad b_{3}=0.5, \quad e_{3}=0.5, \quad \gamma_{3}=0.01, \quad \delta_{3}=0.5, \quad \varepsilon_{3}=0.5 \text {, } \\
& r_{4}=1.02, \quad b_{4}=0.5, \quad e_{4}=0.5, \quad \gamma_{4}=0.01, \quad \delta_{4}=0.49, \quad \varepsilon_{4}=0.5 \text {, } \\
& r_{5}=1.01, \quad b_{5}=0.5, \quad e_{5}=0.5, \quad \gamma_{5}=0.02, \quad \delta_{5}=0.5, \quad \varepsilon_{5}=0.5 \text {, } \\
& r_{6}=1.01, \quad b_{6}=0.5, \quad e_{6}=0.5, \quad \gamma_{6}=0.01, \quad \delta_{6}=0.5, \quad \varepsilon_{6}=0.5, \\
& r_{7}=1.01, \quad b_{7}=0.5, \quad e_{7}=0.5, \quad \gamma_{7}=0.01, \quad \delta_{7}=0.5, \quad \varepsilon_{7}=0.5 \text {, } \\
& r_{8}=1.02, \quad b_{8}=0.5, \quad e_{8}=0.5, \quad \gamma_{8}=0.01, \quad \delta_{8}=0.49, \quad \varepsilon_{8}=0.5 \text {. }
\end{aligned}
$$

Assume

$$
\begin{aligned}
& d_{12}^{y x}=d_{14}^{y x}=d_{23}^{y x}=d_{34}^{y x}=d_{67}^{y x}=d_{78}^{y x}=d_{56}^{y x}=d_{58}^{y x}=0.01, \\
& d_{21}^{x y}=d_{32}^{x y}=d_{41}^{x y}=d_{43}^{x y}=d_{53}^{x y}=d_{65}^{x y}=d_{76}^{x y}=d_{85}^{x y}=d_{87}^{x y}=-0.01 .
\end{aligned}
$$


Otherwise,

$$
d_{i j}^{x y}=d_{i j}^{y x}=0 .
$$

Then, we can obtain

$$
d_{i j}^{y x}=0 \quad(i>j), \quad d_{i j}^{x y}=0 \quad(i \leq j) .
$$

The cross-dispersal matrix is listed as follows:

$$
\begin{aligned}
R & =\left(\left|\beta_{i j}\right|\right)_{n \times n} \\
& \left(\begin{array}{cccccccc}
0 & e_{1}\left|d_{12}^{y x}\right| & 0 & e_{1}\left|d_{14}^{y x}\right| & 0 & 0 & 0 & 0 \\
\varepsilon_{2}\left|d_{21}^{x y}\right| & 0 & e_{2}\left|d_{23}^{y x}\right| & 0 & 0 & 0 & 0 & 0 \\
0 & \varepsilon_{3}\left|d_{32}^{x y}\right| & 0 & e_{3}\left|d_{34}^{y x}\right| & 0 & 0 & 0 & 0 \\
\varepsilon_{4}\left|d_{41}^{x y}\right| & 0 & \varepsilon_{4}\left|d_{43}^{x y}\right| & 0 & 0 & 0 & 0 & 0 \\
0 & 0 & \varepsilon_{5}\left|d_{53}^{x y}\right| & 0 & 0 & e_{5}\left|d_{56}^{y x}\right| & 0 & e_{5}\left|d_{58}^{y x}\right| \\
0 & 0 & 0 & 0 & \varepsilon_{6}\left|d_{65}^{x y}\right| & 0 & e_{6}\left|d_{67}^{y x}\right| & 0 \\
0 & 0 & 0 & 0 & 0 & \varepsilon_{7}\left|d_{76}^{x y}\right| & 0 & e_{7}\left|d_{78}^{y x}\right| \\
0 & 0 & 0 & 0 & \varepsilon_{8}\left|d_{85}^{x y}\right| & 0 & \varepsilon_{8}\left|d_{87}^{x y}\right| & 0
\end{array}\right) .
\end{aligned}
$$

Simple computation results in

$$
R=\left(\begin{array}{cccccccc}
0 & 0.005 & 0 & 0.005 & 0 & 0 & 0 & 0 \\
0.005 & 0 & 0.005 & 0 & 0 & 0 & 0 & 0 \\
0 & 0.005 & 0 & 0.005 & 0 & 0 & 0 & 0 \\
0.005 & 0 & 0.005 & 0 & 0 & 0 & 0 & 0 \\
0 & 0 & 0.005 & 0 & 0 & 0.005 & 0 & 0.005 \\
0 & 0 & 0 & 0 & 0.005 & 0 & 0.005 & 0 \\
0 & 0 & 0 & 0 & 0 & 0.005 & 0 & 0.005 \\
0 & 0 & 0 & 0 & 0.005 & 0 & 0.005 & 0
\end{array}\right) .
$$

After a calculation, we obtain the positive equilibrium for system (12) as

$$
E^{*}=(1,1,1,1, \ldots, 1,1) .
$$

Based on Fig. 2, we know that the diagraph $(G, A)$ is not strongly connected. However, it has two strongly connected components with two layers. Using Corollary 3.4, we obtain that the positive equilibrium point $E^{*}$ of system (12) is globally asymptotically stable in $R_{+}^{2 n}$.

\section{Conclusions and outlooks}

In this paper, cross-dispersal is considered in the predator-prey model with a patchy environment. A new predator-prey model with cross-dispersal among patches is constructed. A new cross-dispersal matrix is established by the coupling relationship between vertices. Based on a graph-theoretical approach for coupled systems and constructing Lyapunov functions, sufficient conditions that the positive equilibrium of the new model is globally 


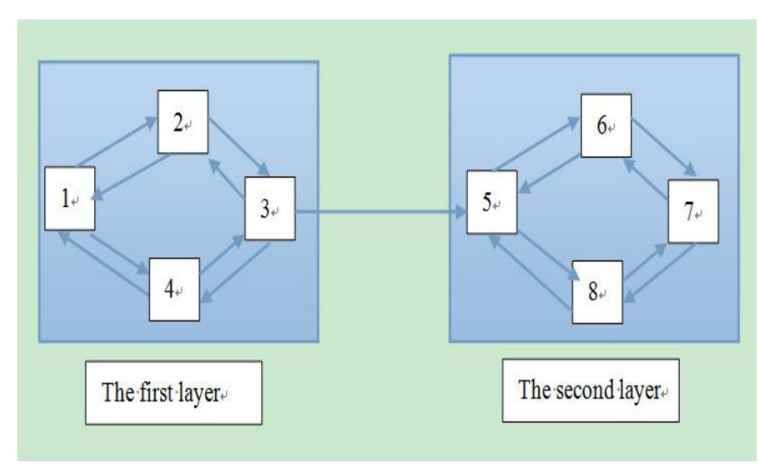

Figure 2 Strongly connected components

stable are derived on a network with strongly connected graphs. Furthermore, based on the theory of asymptotically autonomous systems and the hierarchical method in graph theory, a stability theorem for the positive equilibrium is established on a complex network without strongly connected graphs. Two examples are given to illustrate the main results.

The new predator-prey model with cross-dispersal among patches can be seen as a coupled system with complicated coupling relationship. The complicated coupling relationship considered here is very interesting. To the best of the author's knowledge, the strongly connected character and Lemma 2.1 are critical factors in the study for coupled systems of differential equations on networks. Strongly connected graphs and strongly connected components are different (see Example 4.1 and Example 4.2), factually. Therefore, Theorem 3.2 and Theorem 3.3 are both useful in reality.

Parameters $d_{i j}^{x y}$ and $d_{i j}^{y x}$ can be selected and controlled by a cross-dispersal matrix, effectively. If $d_{i j}^{x y}$ and $d_{i j}^{y x}(i>j)$ are chosen such that $\varepsilon_{i}\left|d_{i j}^{x y}\right| \geq e_{i}\left|d_{i j}^{y x}\right|$, then $d_{j i}^{x y}, d_{j i}^{y x}$ can be chosen based on $e_{j}\left|d_{j i}^{y x}\right| \geq \varepsilon_{j}\left|d_{j i}^{x y}\right|$. Furthermore, conditions $H_{1}$ and $H_{2}$ will be checked to adjust the parameters $d_{i j}^{x y}$ and $d_{i j}^{y x}$.

Further studies on this subject are being carried out by this author in two aspects [1923]: one is to study the model with a delay effect; the other is to discuss the model with time-varying parameters.

Acknowledgements

Not applicable.

Funding

This work was supported by the Natural Science Foundation of HeiLongJiang Province (No. LH2020A017).

Availability of data and materials

Not applicable.

\section{Declarations}

Competing interests

The authors declare that they have no competing interests.

Authors' contributions

YG carried out the work in this paper and drafted the manuscript. The author read and approved the final manuscript. 


\section{Publisher's Note}

Springer Nature remains neutral with regard to jurisdictional claims in published maps and institutional affiliations.

Received: 17 February 2021 Accepted: 11 August 2021 Published online: 24 November 2021

\section{References}

1. Zhang, C.H., Shi, L.: Graph-theoretic method on the periodicity of coupled predator-prey systems with infinite delays on a dispersal network. Physica A 561, 125255 (2021)

2. Li, M.Y., Shuai, Z.S.: Global-stability problem for coupled systems of differential equations on networks. J. Differ. Equ. $248(1), 1-20(2010)$

3. Zhang, C.H., Guo, Y., Chen, T.R.: Graph-theoretic method on the periodicity of multipatch dispersal predator-prey system with Holling type-II functional response. Math. Methods Appl. Sci. 41(9), 1-12 (2018)

4. Huang, R., Wang, Y.S., Wu, H.: Population abundance in predator-prey systems with predator's dispersal between two patches. Theor. Popul. Biol. 135, 1-8 (2020)

5. Sun, G.W., Mai, A.L.: Stability analysis of a two-patch predator-prey model with two dispersal delays. Adv. Differ. Equ. 2018, 373 (2018). https://doi.org/10.1186/s13662-018-1833-2

6. Gao, Y., Liu, S.Q.: Global stability for a predator-prey model with dispersal among patches. Abstr. Appl. Anal. 2014, $176493(2014)$

7. Chen, T.R., Sun, Z.Y., Wu, B.Y.: Stability of multi-group models with cross-dispersal based on graph theory. Appl. Math. Model. 47, 745-754 (2017)

8. Guo, Y., Li, Y.J., Ding, X.H.: On input-to-state stability for stochastic multi-group models with multi-dispersal. Appl. Anal. 96(16), 2800-2817 (2017)

9. Guo, Y., Li, Y.W., Ding, X.H.: Razumikhin method conjoined with graph theory to input-to-state stability of coupled retarded systems on networks. Neurocomputing 267, 232-240 (2017)

10. Guo, Y., Zhao, W., Ding, X.H.: Input-to-state stability for stochastic multi-group models with multi-dispersal and time-varying delay. Appl. Math. Comput. 343, 114-127 (2019)

11. Guo, Y., Wang, Y.D., Ding, X.H.: Global exponential stability for multi-group neutral delayed systems based on Razumikhin method and graph theory. J. Franklin Inst. Eng. Appl. Math. 355(6), 3122-3144 (2018)

12. Liu, Y., Mei, J.L., Li, W.X.: Stochastic stabilization problem of complex networks without strong connectedness. Appl. Math. Comput. 332, 304-315 (2018)

13. Meng, X., Kao, Y.G., Karimi, H.R., Gao, C.C.: Global Mittag-Leffler stability for fractional-order coupled systems on network without strong connectedness. Sci. China Inf. Sci. 63, 1-11 (2020)

14. Tan, Y.X.: Dynamics analysis of Mackey-Glass model with two variable delays. Math. Biosci. Eng. 17(5), 4513-4526 (2020)

15. Long, X.: Novel stability criteria on a patch structure Nicholson's blowflies model with multiple pairs of time-varying delays. AlMS Math. 5(6), 7387-7401 (2020)

16. Manickam, I., Ramachandran, R., Rajchakit, G., Cao, J.D., Huang, C.X.: Novel Lagrange sense exponential stability criteria for time-delayed stochastic Cohen-Grossberg neural networks with Markovian jump parameters: a graph-theoretic approach. Nonlinear Anal., Model. Control 25(5), 726-744 (2020)

17. Cao, Q., Long, X: New convergence on inertial neural networks with time-varying delays and continuously distributed delays. AIMS Math. 5(6), 5955-5968 (2020)

18. Hassan, K.K.: Nonlinear Systems, 3rd edn. Prentice Hall, New York (2002)

19. Zhang, X., Hu, H.: Convergence in a system of critical neutral functional differential equations. Appl. Math. Lett. 107, $106385(2020)$

20. Wei, Y., Yin, L., Long, X.: The coupling integrable couplings of the generalized coupled Burgers equation hierarchy and its Hamiltonian structure. Adv. Differ. Equ. 2019, 58 (2019). https://doi.org/10.1186/s13662-019-2004-9

21. Huang, C., Yang, L., Cao, J.: Asymptotic behavior for a class of population dynamics. AlMS Math. 5(4), 3378-3390 (2020)

22. Huang, C., Long, X., Cao, J.: Stability of antiperiodic recurrent neural networks with multiproportional delays. Math. Methods Appl. Sci. 43(9), 6093-6102 (2020)

23. Zhang, H., Qiao, C.F.: Convergence analysis on inertial proportional delayed neural networks. Adv. Differ. Equ. 2020, 277 (2020). https://doi.org/10.1186/s13662-020-02737-3

\section{Submit your manuscript to a SpringerOpen ${ }^{\circ}$ journal and benefit from:}

- Convenient online submission

- Rigorous peer review

- Open access: articles freely available online

- High visibility within the field

- Retaining the copyright to your article

Submit your next manuscript at $\gg$ springeropen.com 